\title{
26. SOME NEW OBSERVATIONS RELATING TO GALACTIC RADIO SOURCES AND BACKGROUND STRUCTURE
}

\author{
E. R. HILL \\ CSIRO Radiophysics Laboratory, Sydney
}

A survey of the distribution of radio emission at wavelengths of 75 and $20 \mathrm{~cm}$ along an extensive section of the Southern Milky Way has recently been carried out by M. Komesaroff and myself using the 210-foot radio telescope at Parkes. The area surveyed ranges from $l \mathrm{II}=280$ to $355^{\circ}$ and extends on the average to 6 degrees either side of the plane. The beamwidths of the aerial at 75 and $20 \mathrm{~cm}$ are 50 and $14 \mathrm{~min}$ arc respectively. Observations at the shorter wavelength offer a picture of this section of the Galaxy at considerably higher angular resolution than hitherto available, while the $75-\mathrm{cm}$ observations are expected to make a useful contribution to our spectral information.

Reduction of our data is most advanced for the 20 -cm observations directed towards the innermost parts of the Galaxy, i.e. between $l \mathrm{II}=315$ and $355^{\circ}$. This region has now been mapped in right ascension and declination, and a small portion in $l^{\mathrm{II}}$ and $b^{\mathrm{II}}$ as well.

Within this particular region of the survey the distribution of 20 -cm wavelength radiation is extremely complex. A large number of new sources have been detected and many features close to the galactic ridge and appearing extended in lower angular resolution surveys have been to some extent resolved into groups of sources.

The analysis of these source data as well as of the unresolved background is still in progress and it is therefore proposed to limit the present discussion primarily to some comments on source identifications and source groupings. A search for groups of sources is of particular interest to galactic structure studies since some source groupings may originate in large complexes of HII regions in various spiral arms, while others may be related to the tangential direction of spiral arms, since in these directions sources stretching out behind one another along quite great lengths of spiral arm would appear as groupings. In connection with the latter it should be remembered that Mills (1959) has suggested that steps in the distribution of continuum emission along the galactic ridge might also indicate tangential points of spiral arms.

Within the longitude range presently under discussion, just over 100 sources having flux densities in excess of about $3 \times 10^{-26} \mathrm{~W} \mathrm{~m}^{-2}(\mathrm{c} / \mathrm{s})^{-1}$ at $20 \mathrm{~cm}$ have been detected within the $3^{\circ} \mathrm{K}$ antenna temperature contours stretched out either side of the galactic plane. Only a very preliminary search has been made for sources on our records but outside these contours however there appear to be very few having flux densities exceeding 5 flux units. On the basis of source counts at higher galactic latitudes, only about half a dozen extragalactic sources with flux density $>3$ flux units are expected in the 400 square degrees of sky here considered. The great majority of our sources are therefore galactic. 
Generally speaking, we have very little information regarding the thermal or nonthermal character of radio emission from most of the sources detected. This state of affairs arises out of the complexity of the distribution of radiation revealed by the present survey and from the low angular resolution of the low-frequency measurements already available for this region of sky, e.g. at $85 \mathrm{Mc} / \mathrm{s}$. However, there are some sources which are sufficiently isolated to allow good estimates of their spectrum to be obtained; one such case is MSH 14-63 discussed below. Other sources identified with HII regions may be considered to be thermal.

\section{Identifications}

Attempts have been made to identify sources with HII regions listed by Rodgers, Campbell, and Whiteoak (1960) in their Table II. Between $l^{\mathrm{II}}=315$ and $331^{\circ}$ they list only five HII regions owing to the presence of local obscuration; two can be

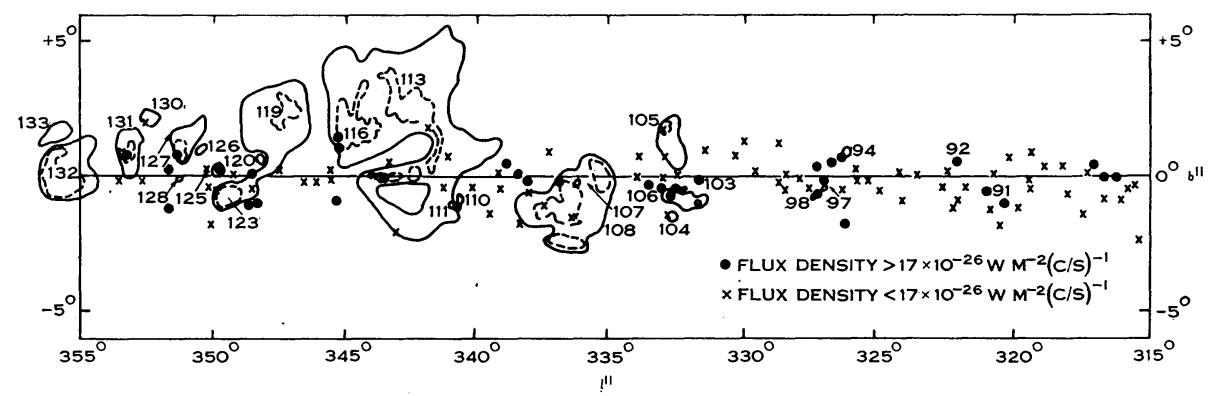

Fig. 1.-The distribution of sources (dots and crosses) observed at 20-cm wavelength between the $3^{\circ} \mathrm{K}$ antenna temperature contours lying on each side of the galactic ridge is shown superimposed on the outline of HII regions (numbered), taken from the Mount Stromlo Atlas of $\mathrm{Ha}$ emission.

definitely identified as sources. From $l \mathrm{II}=331$ to $355^{\circ}$ many more HII regions are visible and most of the brighter and larger regions are clearly radio sources. Even some of the more extensive features, particularly those extending away from the galactic plane, are clearly related to similar features in the radio distribution. Figure 1 shows the radio source positions superimposed on a map of the HII region distribution taken from the Mount Stromlo Atlas (Rodgers et al. 1960).

The majority of sources still remain unidentified as yet; one exception is MSH 14-63 lying near $l \mathrm{II}=316^{\circ}, b^{\mathrm{II}}=-2^{\circ}$. Contours of antenna temperature over this source (Fig. 2) show it to be almost a complete ring, approximately 45 min arc diameter. Combining 85 and $1410 \mathrm{Mc} / \mathrm{s}$ observations shows that its spectral index is about -0.4 , i.e. $S \propto f^{-0.4}$. The nonthermal radio spectrum and the shelllike appearance of the source suggest that it is a supernova remnant and that faint filamentary nebulosity usually found with such remnants might be detected. A search for such filaments has been made by Westerlund and he will report his findings later. Further optical studies of other sources somewhat similar in radio appearance to 14-63 are under way. 


\section{Source Groupings}

The stronger sources detected in the present survey show definite signs of grouping. This is seen in Figure 1 where the sources whose flux density is greater than about $17 \times 10^{-26} \mathrm{~W} \mathrm{~m}^{-2}(\mathrm{c} / \mathrm{s})^{-1}$, indicated by the filled-in circles, form groups at $l \mathrm{II}=316,326,333$, and $338^{\circ}$. Between $l \mathrm{II}=343$ and $355^{\circ}$ there are no obvious groupings of the stronger sources. With the exception of the one at $l \mathrm{II}=326^{\circ}$, the

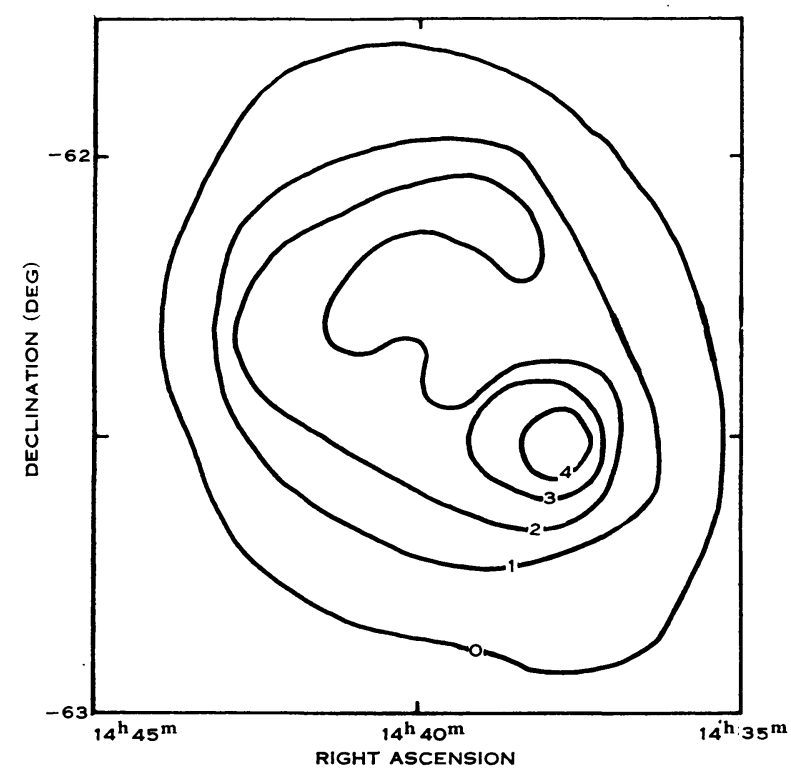

Fig. 2.-Contours of antenna temperature in ${ }^{\circ} \mathbf{K}$ at 20-cm wavelength, of the galactic nonthermal source 14-63 which is believed to be a supernova remnant.

groups are seen clearly in the distribution of antenna temperature along the great circle $b^{\mathrm{II}}=0$ (Fig. 3). The $l^{\mathrm{II}}=326^{\circ}$ group is more spread in galactic latitude than the others and this explains its comparative insignificance in Figure 3.

In general the source groupings comprise between three and five strong sources and occupy an area of about 3 square degrees. They appear to sit on humps which may be constituted of somewhat fainter unresolved sources associated with the groups. So far it has not been possible to make any clear-cut identifications of any members of the groups.

The grouping at $l \mathrm{II}=326^{\circ}$ is distinguished from the others not only as a result of a greater spread in latitude but also because it occurs at a step in the background distribution. A step also appeared in the $85 \mathrm{Mc} / \mathrm{s}$ survey at this point and was attributed by Mills (1959) to the onset of nonthermal radiation associated with a spiral arm. Later Mathewson, Healey, and Rome (1962), from a comparison of their survey at $1440 \mathrm{Mc} / \mathrm{s}$ and the $85 \mathrm{Mc} / \mathrm{s}$ survey, concluded that the step at both frequencies at this longitude resulted from an enhanced belt of ionized interstellar medium surrounding the galactic centre. 
A contour diagram of the $l \mathrm{II}=326^{\circ}$ region appears in Figure 4 . Here the background step is seen to set in rather rapidly at $l \mathrm{II}=325.9$ and $b^{\mathrm{II}}=-0.5$. The width (between half-intensity points) of this enhanced emission in galactic latitude is only about $0: 8$. In addition to this step and the source cluster, there also appears to be an enhancement of a wider latitude spread as indicated by the dispersal of the lower antenna temperature contours. Thus, in this neighbourhood of the galactic plane, three new components make their appearance.

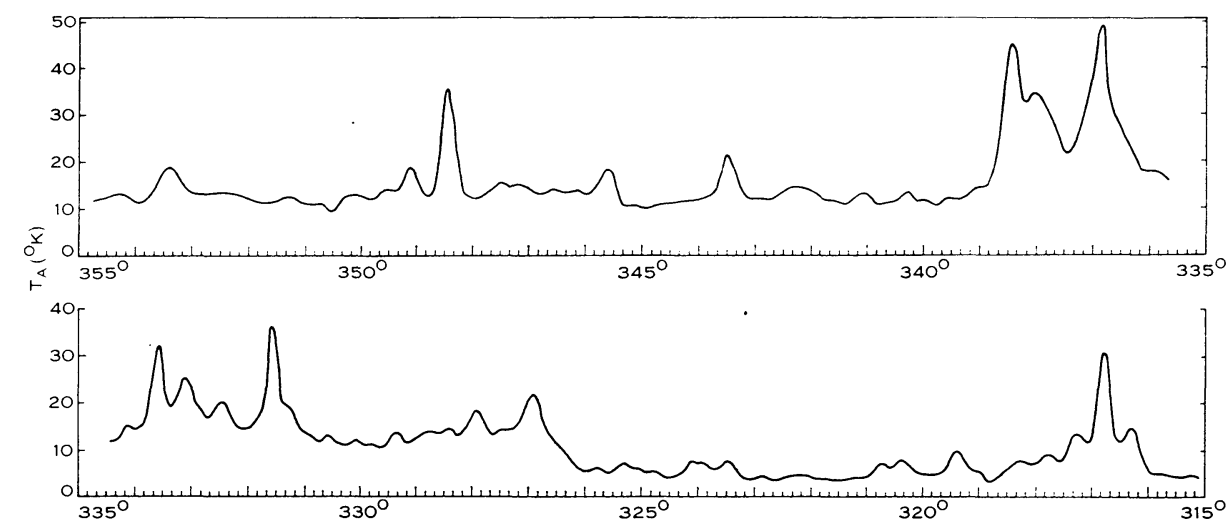

Fig. 3. - The distribution of antenna temperature in ${ }^{\circ} \mathrm{K}$ along portion of the great circle $b^{\text {II }}=0$.

It is the H-line observations which probably reveal the underlying cause for the peculiarities noted in the continuum emission in this region. According to the interpretation of $\mathrm{H}$-line observations in the Southern Milky Way recently proposed by Kerr (1962), it would appear that close to $l \mathrm{II}=326^{\circ}$ we are looking tangentially along a distant spiral arm. This arm has been called the Scutum-Norma arm and in this direction it extends between distances 4 and $8 \mathrm{kpc}$ from the Sun. The source grouping and the background components entering into the general distribution of continuum radiation at this point are presumably related to this arm. If this is the case then the sources noted, if thermal, are extremely intense, being comparable with the Omega nebula. The emission associated with the step would correspond to a width of about $80 \mathrm{pc}$ perpendicular to the galactic plane. The other background component would stretch somewhat further either side of the plane; just how far can be determined only when the data at more outlying galactic latitudes have been analysed.

The other source groupings, those at $l \amalg=316,333$, and $338^{\circ}$, would not appear to highlight tangential directions to spiral arms. Apart from the fact that their spread in galactic longitude suggests that they are stretched out along spiral arms crossing the line of sight, there are no pronounced steps in the background temperature distribution associated with them, nor is there evidence at present of a wider component being introduced with them as with the $l \mathrm{II}=326^{\circ}$ grouping. Finally there is no evidence supporting this interpretation from $\mathrm{H}$-line data. A more attrac- 
tive explanation for these groupings is that they represent large-scale concentrations of ionized material, related possibly to stellar associations connected with the Sagittarius or Scutum-Norma spiral arms.

The evidence presented here relating one of the four source groupings and background structure apparently related with it to the tangential direction to a spiral arm, and the other source groupings to large-scale concentrations of ionized matter

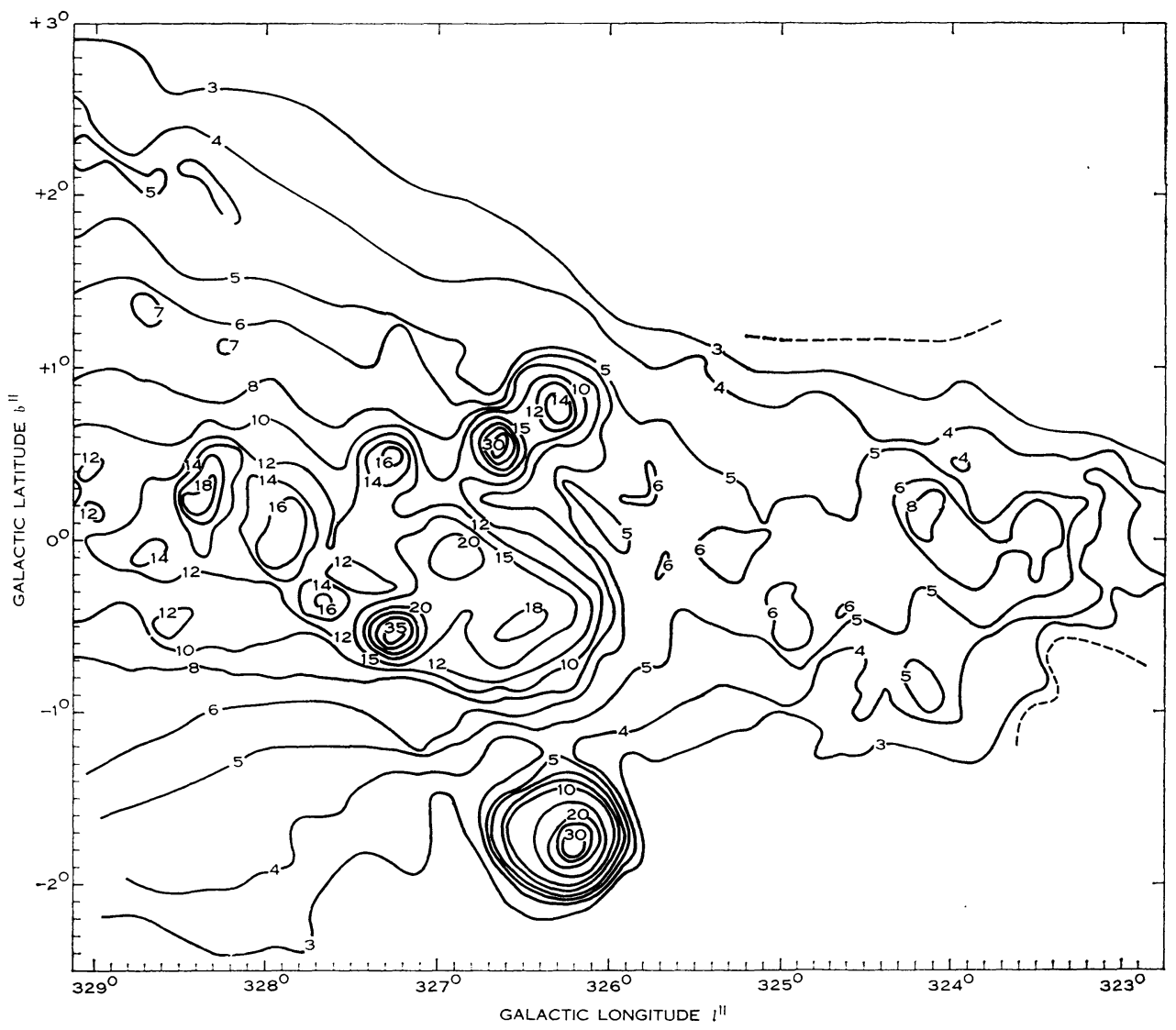

Fig. 4. - Contours of antenna temperature in ${ }^{\circ} \mathrm{K}$ in the vicinity of $l \mathrm{II}=326^{\circ}$, i.e. around the tangential direction to the Scutum-Norma spiral arm.

in other arms, can at present only be regarded as highly suggestive. It is clearly of considerable importance to pursue these continuum investigations at other frequencies and over a more extensive range of galactic longitude. For in conjunction with $\mathrm{H}$-line observations it should be possible to determine the connection between thermal and nonthermal galactic sources as well as groups of such sources, and spiral structure, over a quite extensive region of the Galaxy. Furthermore, such studies, particularly near well-established directions of spiral arm tangents, should also allow a determination of the extent to which the galactic disk background originates in the spiral arms of the Galaxy. 


\section{References}

KERR, F. J. (1962).-M.N. 123: 327-45.

Mathewson, D. S., Healey, J. R., and Rome, J. M. (1962).-Aust. J. Phys. 15 : 369-77.

MiLLS, B. Y. (1959).-P.A.S.P. 71 : 267-91.

Rodgers, A. W., Campbell, C. T., and Whiteoak, J. B. (1960).-M.N. 121 : 103-10.

Rodgers, A. W., CAmpbell, C. T., Whiteoak, J. B., Batley, H. H., and Hunt, V. O. (1960).-

"An Atlas of H-alpha Emission in the Southern Milky Way." (Mount Stromlo Observatory:

Canberra.)

\section{Discussion}

Bok: We should not overlook the possibility that some of Mr. Hill's features may be related to relatively nearby optical features. Tomorrow I shall present evidence for the presence of an OB grouping near $l \mathrm{II}=315^{\circ}$ at an average distance of $1800 \mathrm{pc}$, with an overlying absorption of 1.5 to 2 mag. Near $l^{11}=327^{\circ}$, we find two concentrations, one at a distance of $870 \mathrm{pc}$, the other at 2400 pc from the Sun. Neither of these seems to be in the Norma-Scutum arm. Rather do they appear to be along an edge of the Sagittarius arm.

Hill: It is perfectly true that a number of the 20 -cm continuum sources I have spoken of may well be relatively nearby. It appears equally true, however, that others are likely to be quite distant-for example, the sources near $l \mathrm{II}=326^{\circ}$. Future $\mathrm{H}$-line absorption studies of these sources should provide a solution to these distance problems. It should be added that we can detect, at 20 -cm wavelength, HII regions like the Omega nebula even when they are at distances of the order of $6 \mathrm{kpc}$.

Thackeray: Could I ask Mr. Hill to expand his remarks a little on what are the points of similarity or dissimilarity between his various longitude groups?

Hill: The principal differences between the various longitude groupings of our sources relate to some extent to the latitude distribution of the sources in the groups, one group at $l \mathrm{II}=326^{\circ}$ showing noticeably more spread in galactic latitude than the other groups which appear to be stretched out in galactic longitude. However, the greatest distinction between the $l \mathrm{II}=326^{\circ}$ region and the others relates to the distinct step in the ridge temperatures found at this longitude, both at $85 \mathrm{Mc} / \mathrm{s}$ and $1410 \mathrm{Mc} / \mathrm{s}$. At $l \mathrm{II}=308^{\circ}$ for instance, a step appears only at $85 \mathrm{Mc} / \mathrm{s}$; Mathewson, Healey, and Rome found no step at this longitude at $1440 \mathrm{Mc} / \mathrm{s}$.

Westerhout: What is the longitude extent of this bulge? Does it come back again to a narrow latitude distribution at higher longitudes?

Hill: The data have not been sufficiently reduced yet to give an explicit answer to this question. My initial impression is, however, that the narrow latitude distribution is not met again at the higher longitudes.

Lequeux: Observations on 21 and $13 \mathrm{~cm}$ with the large radio telescope in Nançay support strongly Hill's observations of grouping of thermal radio sources. Interferometric observations of thermal sources at Cal Tech and Nançay show that many have some important fine structure, for which a characteristic dimension is $1 \mathrm{pc}$; we need for complete study of HII regions a resolving power of about 1 minute of arc, if possible given by a pencil-beam instrument.

Davies: At Jodrell Bank, Dr. Hazard and I made a survey of the anticentre region of the Galaxy at $237 \mathrm{Mc} / \mathrm{s}$ which I have used to compare with the Sky Atlas plates. Three radio features were found to coincide in position and extent with optical shell-like nebulosities and four were thought to be probably associated with objects also thought to be supernova remnants. The spectrum of the radio objects and the shape of the optical object were used as criteria to distinguish the supposed supernova remnants from HII regions.

Oort: Do you make any distinction between the thermal and nonthermal sources?

Hill: At present it is generally not possible to determine the thermal or nonthermal character of most of the sources from their radio spectrum because of the difficulty in identifying them with sources found at other frequencies, e.g. at $85 \mathrm{Mc} / \mathrm{s}$. The complexity of the distribution of radiation found in the present survey at $20 \mathrm{~cm}$ and the lower angular resolution of surveys 
of this region at other frequencies bring about this state of affairs. Some progress can, however, be made along these lines in certain comparatively featureless regions. MSH 14-63 mentioned above is such a case, since it lies in a comparatively structureless area about 2 degrees south of the galactic plane. In other cases where a source can be identified with an HII region it may be presumed to be thermal.

Oort: There appears to be little concentration of the sources to the galactic centre.

Hill: This is so; the only change noted as one nears $l \mathrm{II}=0$ is the more uniform longitude distribution of the brighter sources as opposed to the clumping present further from the direction of the galactic centre.

Mathewson: Separation of the disk radiation into thermal and nonthermal components by using the $1440 \mathrm{Mc} / \mathrm{s}$ survey of Mathewson, Healey, and Rome and the $85.5 \mathrm{Mc} / \mathrm{s}$ survey of Hill, Slee, and Mills (both surveys had $50 \mathrm{~min}$ of arc aerial beams) has shown the existence of a strong central thermal component which in the Southern Milky Way commences at $l^{\mathrm{II}}=327^{\circ}$. It is extremely interesting to see that this component is not resolved into discrete sources by the $14 \mathrm{~min}$ beam of the 210 -foot reflector at $1410 \mathrm{Mc} / \mathrm{s}$. This suggests that this central thermal component is indeed a large-scale feature of the Galaxy.

Westerhout: It is interesting to see that the bulge in the Norma-Scutum arm to higher latitudes is some sort of wide component suddenly added. Thus in this region one has an increase in the wide nonthermal distribution, a step in the thermal distribution, and an increase in number of sources.

Hill: Yes, this seems to be so, although we will have to examine in more detail the separation of the unresolved background into its thermal and nonthermal components before we can be quite sure of this interpretation.

Komesaroff: I think from memory at this longitude there is a step in the Mills $85 \mathrm{Mc} / \mathrm{s}$ contours which coincides roughly with the step in the thermal component found by Mathewson, Healey, and Rome, suggesting that both components rise at this point.

Parijsky: Is it possible to find any region completely free from discrete sources? If it is possible we may apply a source statistical method for obtaining the fine structure of the Milky Way, such as Scheuer proposed for the extragalactic sources.

Hill: Within the longitude range here considered, there are areas in the disk that are apparently free of sources, but they are not very extensive. Possibly that part of our survey still not analysed would provide more opportunities for such an analysis.

Mills: I would just like to make the obvious point that one does not expect both thermal and nonthermal radiation to be distributed uniformly along spiral arms. Consequently sometimes spiral arm directions might be indicated by thermal and sometimes by nonthermal radiation. It is only the $327^{\circ}$ arm that appears to show both strongly together with the other component, the discrete sources.

Mathewson: You comment on the increase of nonthermal radio emission at $l^{\mathrm{II}}=327^{\circ}$ as shown by the Hill, Slee, and Mills $85 \mathrm{Mc} / \mathrm{s}$ contours. I would like to point out that this could be due to the increase in the thermal component of the radiation at this point which would still make an appreciable contribution even at the low frequency of $85 \cdot 5 \mathrm{Mc} / \mathrm{s}$.

Hill: The separation of thermal and nonthermal background components in this region will have to be re-examined, particularly since we are now better able to separate out the effects of the isolated sources.

Kerr: Absorption observations give a possible way of obtaining distance estimates for many of these sources, or at least of comparing the relative positions of line and continuum sources. Absorption effects have been seen in the 210-foot hydrogen line observations in the position of all the sources along the galactic equator.

The "step" at $l^{\mathrm{II}}=327^{\circ}$ shows up also in the integrated line brightness, and this is the only significant step in integrated brightness.

Bok: Radio astronomers should not plan to place too much weight on the edges found from the work of Elsässer and Haug. Without further study one cannot decide to what degree inter- 
stellar absorption affects the observed distributions. If, optically, one desires to list directions for which one is most likely looking along a spiral arm-then one can do so only on the basis of exhaustive regional surveys based on spectra, $U B V$ colours, absolute magnitude estimates, and radial velocities.

Westerlund: The possible supernova remnant at $14^{\mathrm{h}} 38^{\mathrm{m}},-62^{\circ} 30^{\prime}$ has been photographed in red light with the Uppsala telescope. The apparent centre of the brightest arc (RCW No. 86) is about $4^{\prime}$ from the peak-temperature $\left(4 \cdot 8^{\circ} \mathrm{K}\right)$ point of the radio source. Another weak arc follows exactly the northern $2^{\circ} \mathrm{K}$ contour. The inside of the first arc is rich in filaments and one filament goes through the $4 \cdot 8^{\circ} \mathrm{K}$ point. The length of the most pronounced arc is $\sim 5^{\prime}$. The structure of the filaments does not indicate an ordinary HII region. We hope to obtain a spectrum of the brightest filament with our nebular spectrograph.

\section{OBSERVATIONS DE RESTES DE SUPERNOVAE A LA STATION DE RADIOASTRONOMIE DE NANÇAY}

\section{A. Boischot et J. Lequeux \\ Observatoire de Paris-Meudon}

La structure de restes de supernovae galactiques de petit diamètre apparent a été étudiée sur $1420 \mathrm{Mc} / \mathrm{s}$ avec l'interféromètre à 2 antennes de Nançay, dont la résolution maximale est $18^{\prime \prime} \mathrm{EW}$ et $\mathrm{l}^{\prime} \mathrm{NS}$ (Lequeux 1962). Taurus A a des dimensions de $3^{\prime}, 9 \times 2^{\prime}, 7$ à demi-intensité, l'angle de position du grand axe étant $126^{\circ}$. L'émissivité dans cette radiosource croît rapidement vers le centre; d'autre part ses dimensions augmentent avec la longueur d'onde, ce qui peut s'expliquer d'après Woltjer (1958) par une création continue d'électrons relativistes au centre de la radiosource. Cassiopeia A, au contraire de Taurus A, est parfaitement circulaire; en admettant la symétrie sphérique, nos observations montrent que presque toute l'émission radioélectrique provient d'une enveloppe sphérique de $2^{\prime}, 0$ de rayon et $0^{\prime}, 6$ d'épaisseur à demi émissivité. Les résultats obtenus sur les Supernovae de Képler et Tycho Brahé sont en accord avec ceux de Cal Tech sur $960 \mathrm{Mc} / \mathrm{s}$ : la Supernova de Tycho Brahé est peut-être une enveloppe sphérique, mais on ne peut rien dire de celle de Képler.

D'autres restes de supernovae de plus grand diamètre ont été étudiés plus récemment avec la partie actuellement construite $(40 \mathrm{~m} \times 35 \mathrm{~m})$ du grand radiotélescope de Nançay, dont le pouvoir de résolution est environ $20^{\prime} \times 20^{\prime}$ à $1430 \mathrm{Mc} / \mathrm{s}$ et $12^{\prime} \times 12^{\prime}$ à $2300 \mathrm{Mc} / \mathrm{s}$. Une carte d'isophotes de HB 21 sur $1430 \mathrm{Mc} / \mathrm{s}$ (Boischot 1962) montre une structure en anneau que l'on retrouve également dans d'autres restes de supernovae (IC 443, HB 9, CTA 1), et dans la Nébuleuse de la Rosette, dont on peut se demander si elle n'est pas aussi un reste de supernova. Sur la carte de HB 21 publiée par Boischot (1962), la déclinaison est erronée et doit être augmentée de $30^{\prime}$. Enfin, W44 et la partie non-thermique de W28 ont été dédoublées en ascension droite sur $2300 \mathrm{Mc} / \mathrm{s}$ et sont sans doute des restes de supernovae en forme d'enveloppe sphérique; la source non-thermique dans W28, dont le diamètre est $28^{\prime}$ et l'indice spectral $-0,37 \pm 0,10$, est identifiée à un anneau de $6^{\prime}$ de diamètre, entouré de fragments d'anneaux concentriques s'étendant sur $24^{\prime}$ de diamètre, bien visibles sur le Sky Atlas (Nguyen Quang 1963). 